\title{
Identifying influences of the quality of interaction on dropout rates of MOOC: preliminary results
}

\author{
Beatriz Brito do Rêgo, Filipe Adeodato Garrido, Ecivaldo de Souza Matos \\ Departamento de Ciência da Computação (DCC) - Instituto de Matemática e Estatística \\ (IME) - Universidade Federal da Bahia (UFBA) \\ Salvador - BA - Brasil \\ \{beatrizbr, filipe.garrido, ecivaldo\}@ufba.br
}

\begin{abstract}
MOOC have emerged as innovation to improve education through the provision of free online courses. However, there is a high drop-out rate for its students. This research is analyzing, from the technological point of view, as possible causes of abandonment. In order to investigate the communication problems of the interface between students on abandonment and withdrawal in MOOC courses, the Semiotic Inspection Method (SIM) was applied, correlating its results with potential causes found in the literature. It was inferred that ruptures of communication are associated with some of the general causes that lead to abandonment or withdrawal.
\end{abstract}

Resumo. Os MOOC surgiram como inovação para melhorar a educação por meio da prestação de cursos online gratuitos. Todavia, registra-se alta taxa de abandono dos seus estudantes. Esta pesquisa está analisando, sob o ponto de vista tecnológico, as possíveis causas de abandono. Para investigar se os problemas de comunicabilidade da interface podem estar relacionados ao abandono e à desistência em cursos MOOC, aplicou-se o Método de Inspeção Semiótica (MIS), correlacionando seus resultados às potenciais causas gerais encontradas na literatura. A partir dos resultados inferiu-se que rupturas de comunicabilidade estão associados com algumas das causas gerais que levam ao abandono ou à desistência.

\section{Introdução}

Os Massive Open Online Courses (MOOC) surgiram como possibilidade de democratização da educação por meio da prestação de cursos online, massivos e gratuitos [He et al., 2015]. Uma das principais críticas aos MOOC está relacionada à baixa taxa de permanência dos estudantes [Grainger, 2013]. Embora milhares de estudantes inscrevam-se a todo momento, a taxa de permanência da maioria dos cursos está em torno de 13\% [Onah et al., 2014]. A literatura aponta algumas "causas-gerais" para esse fenômeno [Gomez-Zermeno \& De La Garza, 2016].

Este artigo apresenta resultados parciais dessa pesquisa, a partir da investigação de como problemas de interação humano-computador, sob o ponto de vista da metacomunicação de interface - rupturas de metacomunicação - podem estar associados ao abandono ou à desistência dos estudantes.

\section{Metodologia}


VI Congresso Brasileiro de Informática na Educação (CBIE 2017)

Anais do XXVIII Simpósio Brasileiro de Informática na Educação (SBIE 2017)

Para realizar esse estudo foi escolhido o curso intitulado Successful Negotiation: Essential Strategies and Skills (Negociações de sucesso: estratégias e habilidades essenciais) da plataforma Coursera ${ }^{1}$. Essa plataforma foi escolhida por apresentar um dos maiores índices de desistência em seus cursos [He et al., 2015; Onah et al., 2014].

Esta fase da pesquisa foi realizada em três etapas. Na primeira etapa buscou-se na literatura por razões-gerais pelas quais estudantes desistem de seus cursos MOOC. Na segunda etapa, utilizou-se o Método de Inspeção Semiótica (MIS) [De Souza \& Leitão, 2009] para encontrar rupturas de comunicabilidade. A presença dessas rupturas indica problemas na metacomunicação de interface, logo, na qualidade da interação. Cenários foram criados, de modo a viabilizar a realização do MIS. Um cenário possui um enredo, que inclui sequências de ações e eventos: o que os usuários fazem o que acontece com eles, que mudanças ocorrem no ambiente.

Os cenários foram produzidos com as seguintes cenas/etapas: (i) cadastro do usuário; (ii) seleção do curso do tipo MOOC; (iii) início do curso; e (iv) participação no fórum, compartilhando informações e experiências ao longo do curso. Além do cenário definido, o MIS foi executado sobre signos metalinguísticos da Central de Ajuda.

Após a inspeção de cada signo, o avaliador reconstituiu a metamensagem correspondente. Por fim, as três mensagens de metacomunicação foram consolidadas e os resultados sintetizados.

$\mathrm{Na}$ terceira e última etapa, os dados obtidos nas duas primeiras etapas (causas-gerais para abandono/desistência e rupturas de comunicabilidade) foram correlacionados em busca de evidências preliminares indicativas de possíveis relações entre a qualidade da metacomunicação de interface e o abandono em cursos MOOC.

\section{Resultados}

$\mathrm{Na}$ inspeção dos signos dinâmicos foi possível visualizar a existência de um signo informando quantos minutos faltavam para o usuário concluir cada módulo. Ao lado dessa informação havia um símbolo, representado por um livro aberto, com a frase "nada expirado". Esse símbolo poderá significar ao estudante que o material do curso possui limitação de tempo. No tocante à relação entre os motivos de abandono e a escassez de tempo, o usuário precisaria se esforçar para conseguir finalizar o módulo antes que o tempo de acesso ao material expire.

Ao iniciar o curso, percebeu-se que a legenda do vídeo não estava sincronizada com as imagens. Para que esse erro fosse corrigido, foi necessário buscar ajuda no símbolo estático que referenciava a imagem de uma bandeira. Associado pelo avaliador como um sinal de socorro. Logo, a depender do perfil do usuário, ele não conseguiria solicitar ajuda para corrigir essa falha de sincronia, havendo insuficiência para o uso das tecnologias.

Outro aspecto confirmado com a avaliação do MIS foram as falhas de tradução na interface, relacionadas aos signos metalinguísticos. O usuário configura o idioma desejado, mas trechos da Plataforma permanecem em outro idioma, nesse caso o inglês,

\footnotetext{
${ }^{1}$ https://www.coursera.org/
} 
VI Congresso Brasileiro de Informática na Educação (CBIE 2017)

Anais do XXVIII Simpósio Brasileiro de Informática na Educação (SBIE 2017)

padrão do Coursera. Caso o usuário não possua familiaridade com a língua inglesa poderá abandonar o curso.

Os itens dificuldade em expor ideias de maneira clara numa comunicação escrita e privação da interação entre aluno e professor não foram notados nas tarefas realizadas pelos avaliadores. O estudante é informado sobre o curso utilizar vídeos previamente gravados, não sendo exibidas aulas ao vivo do professor; portanto, sem interação direta. Ao término dessa etapa da pesquisa, notou-se que a plataforma Coursera disponibiliza diversos atalhos para a central de ajuda; contudo, o inglês é a língua predominante. Caso o usuário não possua domínio no idioma poderá ter dificuldades e desistir do curso.

\section{Considerações Parciais}

Os dados (ainda não conclusivos) mostram que possivelmente falhas na comunicabilidade contribuem para a desistência ou o abandono de estudantes em MOOC, quando consideradas a sua influência na ocorrência das causas-gerais. Embora a maioria das causas tenha sido confirmada pela análise do MIS, será realizada avaliação da metacomunicação com usuários reais, por meio do Método de Avaliação de Comunicabilidade (MAC) [De Souza \& Leitão, 2009]; de modo a confirmar as rupturas identificadas no MIS e, ainda, observar novas rupturas ainda não identificadas.

Para tanto, na próxima fase desta pesquisa realizar-se-á experimentos com porções da mesma plataforma. Será necessário manter o mesmo binômio < plataforma, curso > para evitar ruídos nos dados. Além disso, pretende-se avaliar o processo de design de interação e utilizar técnicas específicas com o intuito de avaliar e prevenir que inconsistências sejam propagadas para a interface.

\section{Agradecimentos}

Agradecemos à Fundação de Amparo à Pesquisa do Estado da Bahia (Fapesb) pelo financiamento de desta pesquisa e ao Grupo de Pesquisa e Extensão em Informática, Educação e Sociedade - Onda Digital - da Universidade Federal da Bahia.

\section{Referências}

De Souza, C. S.; Leitão, C. F. (2009). Semiotic Engineering Methods for Scientifc Research in HCI. Morgan and Claypool, University of Sheffeld, U.K.

Grainger, B. (2013). Introduction to moocs: avalanche, illusion or augmentation. Policy Brief - UNESCO (july). Institute for Information Technologies in Education. URL: http://iite.unesco.org/pics/publications/en/files/3214722.pdf.

Gomez-Zermeno, M. G.; De La Garza, L. A. (2016). Research analysis on mooc course dropout and retention rates. Turkish Online Journal of Distance Education, pages $3-14$.

He, J.; Bailey, J.; Rubinstein, B. I.; Zhang, R. (2015). Identifying at-risk students in massive open online courses. In AAAI, pages 1749-1755.

Onah, D. F.; Sinclair, J.; Boyatt, R. (2014). Dropout rates of massive open online courses: behavioural patterns. EDULEARN14 Proceedings, pages 5825-5834. 